Ann. Biol. anim. Bioch. Biophys., I97o, 10 (3), 473-48o.

\title{
FORME ET VOIE D'ABSORPTION INTESTINALE DES ACIDES GRAS A CHAINE LONGUE CHEZ LA TRUITE ARC-EN-CIEL (SALMO GAIRDNERII RICH.)
}

\author{
II. - LIPIDES " ÉTALÉS " \\ P. BERGOT et J.-E. FLÉCHON \\ Station centrale de Nutrition, \\ Station centrale de Physiologie animale, \\ Centre national de Recherches zootechniques, 78 - Jouy-en-Josas \\ Institut national de la Recherche agronomique

\section{RÉSUMÉ}

L'absorption de l'acide oléique est étudiée en microscopie optique et électronique, dans la muqueuse de l'intestin grêle de Truites arc-en-ciel acclimatées à deux températures différentes $\left(7^{\circ} \mathrm{C}\right.$ et $\left.22^{\circ} \mathrm{C}\right)$. Nous avons observé, dans la cellule absorbante, à côté des chylomicrons, la présence d'une quantité importante de lipides sous une autre forme (lipides " étalés"). Ces lipides sont constitués de triglycérides. Ils ne sont pas entourés par une cytomembrane. Nous les interprétons comme une forme de stockage temporaire des acides gras et non comme une forme d'exportation. Lour présence paraît indépendante de l'état de jeûne et de la température d'acclimatation des animaux. Ces lipides sont également observés pendant l'absorption de l'acide linoléique mais non de l'acide stéarique.

\section{INTRODUCTION}

Dans la cellule intestinale absorbante de Mammifère, où l'absorption des acides gras à chaîne longue se traduit, dans des conditions physiologiques normales, par la formation de chylomicrons, des observations en microscopie électronique ont montré la présence de lipides sous d'autres formes : "stellate lipids" ou "stellate masses of fat " (PALAY et REVEL, I964; FAwCETT, I966), "irregular masses " (TAYLOR et Adamstone, I965), " lipides étalés " (Vodovar et Flanzy, I966) et " free lipid droplets "(CARDEl, SAdenhausen et PORTER, I967).

Chez le Poulet, de grosses gouttelettes lipidiques non entourées par une membrane ont été décrites à côté des chylomicrons (Horman, I968). 
Chez la Truite, nous avons montré (BERGOT et FLÉchoN, I970) que la cellule absorbante exportait les acides gras à chaîne longue, après estérification, sous forme de chylomicrons de petite taille qui ne semblent pas correspondre aux grosses gouttelettes, entourées d'une membrane, décrites chez les alevins de Carassius auratus et Hemiramphus sajori (IWAI, I968; IWAI et TANAKA, I968). Ces grosses gouttelettes ressemblent plutôt à la deuxième forme de lipides déjà signalée chez la Truite, les lipides "étalés" (BERGoT et Vodovar, 1967).

Les observations présentes tentent de préciser la forme et la localisation de ces " lipides étalés" dans la muqueuse intestinale de Truite.

Le matériel et les méthodes utilisées sont les mêmes que précédemment (BERGO'T et FLÉCHON, I970).

\section{OBSERVATIONS}

a) Observations en microscopie photonique sur les coupes à congēlation

Après administration d'acide oléique ou linoléique à des truites à jeun, de grosses gouttelettes soudanophiles de I à $3 \mu$ de diamètre sont présentes dans le cytoplasme des cellules absorbantes de la paroi intestinale. Cependant il est souvent difficile de distinguer ces gouttelettes des accumulations de particules fines dans 1'appareil de Golgi ou les " strutures lamellaires ».

Quoi qu'il en soit, le test du Bleu de Nil indique que dans tous les cas où l'on observe de grosses gouttelettes soudanophiles dans la cellule absorbante, il s'agit de graisses neutres. Les gouttelettes soudanophiles présentes dans certaines cellules de la muqueuse, en particulier les leucocytes de l'épithélium et de la lamina propria, se distinguent des précédentes par leur couleur violacée et non rose.

\section{b) Observations en microscopie électronique}

La présence des lipides "étalés " (LE) est liée à celle des chylomicrons. La taille et la répartition des LE correspondent à celles des grosses gouttelettes de triglycérides observées en microscopie photonique.

Chez les truites acclimatées à $7^{\circ} \mathrm{C}$, les $\mathrm{LE}$ se rencontrent surtout dans la partie supranucléaire des cellules absorbantes, $8 \mathrm{~h}$ après l'administration d'acide oléique à des animaux à jeun (fig. I). On en trouve dans toute la cellule absorbante après I6 $\mathrm{h}$ et principalement dans la partie infranucléaire après $28 \mathrm{~h}$.

Chez les truites acclimatées à $22^{\circ} \mathrm{C}$, les $\mathrm{I}_{1} \mathrm{~F}$, sont déjà présents à ces deux niveaux de la cellule absorbante après $8 \mathrm{~h}$, comme après I $6 \mathrm{~h}$.

On observe également ces LE après fourniture de l'acide oléique non plus seul mais ajouté à la nourriture donnée à des animaux alimentés normalement.

L'acide linoléique donne la même répartition de LE que l'acide oléique. Au contraire, les LE sont totalement absents quand on donne l'acide stéarique à des animaux à jeun. Dans ce cas les seuls lipides visibles dans la cellule absorbante sont des chylomicrons.

Après administration d'acide oléique et double fixation (glutaraldéhyde et tétroxyde d'osmium), ces lipides ont l'aspect de plages circulaires ou ovales, peu opaques aux électrons et délimitées par un contour régulier (fig. 4). 
Après fixation directe par le tétroxyde d'osmitum, les mêmes lipides ont l'aspect de masses opaques aux électrons et de contour irrégulier (fig. 5). Les fixations prolongées par $\mathrm{OsO}_{4}$ (4 heures) ne semblent pas accroître l'opacité aux électrons, mais seulement augmenter l'homogénéité (pas de zone périphérique parfois observée après une fixation de $\mathrm{I} h \mathrm{~h}$ o seulement).

Le contraste des gouttelettes lipidiques n'est pas modifié par le traitement à l'acétate d'uranyle. Il est diminué par le traitement ultérieur au citrate de plomb dans le cas de la double fixation. Cet effet n'a pas été observé après fixation osmique directe.

Le traitement par la thiocarbohydrazide puis $\mathrm{OsO}_{4}$ augmente le contraste entre lipides et tissus fixés par $\mathrm{OsO}_{4}$.

Après fixation osmique simple, la densité aux électrons et l'irrégularité du contour des lipides " étalés" paraissent liées. Elles sont plus importantes :

- chez les truites acclimatées à $7^{\circ} \mathrm{C}$ que chez celles acclimatées à $22^{\circ} \mathrm{C}$, toutes recevant de l'acide oléique (fig. 2 et 5 );

- chez les truites recevant de 1'acide linoléique que chez celles recevant de $1^{\prime}$ acide oléique, toutes étant acclimatées à $7^{\circ} \mathrm{C}$ (fig. 3 et 5 ). Cet aspect ne varie pas aul cours des différentes étapes de 1'absorption observées ( $8 \mathrm{~h}, \mathrm{I} 6 \mathrm{~h}$ ou $28 \mathrm{~h}$ ).

Quand les LE ne sont pas entièrement opaques aux électrons, ils apparaissent entourés par une bordure dense. Cette bordure reste visible autour des $\mathrm{LE}$ séparés du cytoplasme par une dissociation accidentelle de la cellule absorbante après fixation. Elle est également visible autour des LE parvenus en contact (fig. 2 et 6 ). Les images de fusion des $L E$ sont peut fréquentes, ce qui indique un phénomène rare ou rapide.

La bordure dense ne présente pas la structure trilamellaire des membranes plasmiques ou cytoplasmiques. Son épaisseur, que ce soit après simple ou double fixation, apparaît bien inférieure à celle de la membrane cytoplasmique du reticulum endoplasmique. Les LE, s'insérant dans les mailles du réseau formé par les tubules du reticulum endoplasmique apparaissent fréquemment en contact étroit avec ce dernier. Cependant, ce contact s'effectue par la face externe qui porte les ribosomes (fig. 2).

Certaines incidences de coupe permettent d'observer des fibrilles qui entourent partiellement les $L_{E}$ e et donnent l'impression d'une bordure plus épaisse (fig. 6). $L$ 'association des $L_{1} E$ avec les mitochondries ou les membranes de type plasmique des « structures lamellaires » ne se rencontre qu'occasionnellement.

Les $L_{\text {E }}$ se trouvent dans la plus grande partie du cytoplasme de la cellule absorbante ; la zone apicale en est dépourvue. Ils n'ont jamais été observés dans les espaces intercellulaires ni dans le tissu conjonctif sous-épithélial.

\section{DISCUSSION}

Les LE observés chez la Truite présentent les mêmes aspects, compte tenu des effets de la fixation, que les lipides " étalés " et les "free lipids droplets " des cellules absorbantes d'autres vertébrés. Chez les Mammifères, différentes interprétations ont été données. Pour PALAY et Revei, (I964), les "stellate lipids " résulteraient 
soit du passage dans la matrice cellulaire de triglycérides nouvellement synthétisés, le reticulum étant rempli, soit d'un excès de lipides pénétrant trop rapidement dans la cellule pour être pris en charge parle reticulum. Pour Vodovar et Fianzy (I966), les lipides "étalés " seraient la conséquence d'un éclatement de la membrane réticulaire au cours de la préparation des tissus, ou correspondraient à des graisses dont la voie de pénétration n'est pas la même que celle des graisses se trouvant dans la lumière du reticulum. Des lipides sous forme étalée ont aussi été interprétés comme des acides gras à chaîne longue parvenus dans le stroma sans être estérifiés (VoDovar, Flanzy et François, I967).

Chez la Truite, un artefact ne peut expliquer la présence constante des LE après utilisation des différentes techniques. L'interprétation de ces LE comme des acides gras non estérifiés est exclue par le test du Bleu de Nil qui les détermine toujours comme des graisses neutres. Dans le cas présent, il ne peut s'agir non plus de gouttelettes de triglycérides qui auraient pénétré dans la cellule absorbante sous cette forme, car les acides gras ont été donnés à l'état non estérifiés et, de toute façon la pinocý̂́ose paraît négligeable.

Différentes observations (absence des $\mathrm{L}_{1} \mathrm{E}$ à jeun, présence liée à celle des chylomicrons avec l'acide oléique et linoléique, absence avec l'acide stéarique, différences d'aspect suivant l'acide gras) permettent d'attribuer aux acides gras des triglycérides de ces LE une origine essentiellement alimentaire. Des réserves sont cependant à faire sur la correspondance entre l'osmiophilie et le degré d'insaturation des lipides (KORN, I968; SCHIELCHL, I968 ; RIEMERSMA, I968). Malgré cela, on peut penser que la différence d'aspect entre les $L E$ des truites acclimatées à $7^{\circ} \mathrm{C}$ et $22^{\circ} \mathrm{C}$ recevant de 1'acide oléique correspond à un état physico-chimique différent des triglycérides.

SPENCER et al. (I 966$)$ ont fait un rapprochement entre les grosses gouttelettes lipidiques visibles dans l'épithélium intestinal de myxine (Agnathe) après plusieurs semaines de jeûne et les cas d'abêtalipoprotéinémie chez 1'Homme.

Chez la Truite, où l'accumulation de triglycérides dans la cellule absorbante est évidente, la situation paraît différente des cas d'absence congénitale de bêtalipoprotéines (Dobrins, I966) ou des cas d'inhibition de la synthèse protéique chez les Mammifères (SABESIN et ISSELBACHER, I965; ISSELbaCHER, 1967; Vodovar, Flanzy et FrançoIs, I969). En effet, à côté des LE, nous avons toujours observé de nombreux chylomicrons dans le reticulum et l'appareil de Golgi des cellules absorbantes, ainsi que dans les espaces intercellulaires et dans les vaisseaux sanguins et lymphatiques (BERGOT et FLÉCHON, I97O).

Les différents modes d'administration des acides gras expérimentés montrent que les LE sont peut-être dus à la quantité relativement importante d'acides gras ingérés, mais pas à un état pathologique des animaux. IWAI et TaNaka (I968) observent de grosses gouttelettes de plusieurs microns chez un alevin de poisson en alimentation naturelle.

La bordure qui entoure les $\mathrm{LE}$, ressemble à l'interface lipide-eau décrite autour de lipides en émulsion (SchoEFL, I968). L'absence de membrane cytoplasmique autour des LE correspond à ce qui est finalement admis, après controverses, pour les triglycérides des cellules adipeuses (FAWCETT, I964, I966; WoOD, I967).

Dans la cellule hépatique de Mammifère (TrotríR, I967), les triglycérides existent sous deux formes : en grosses gouttelettes et en particules (lipoprotéines). 
Une situation identique nous parait exister dans la cellule absorbante de Truite. Contrairement à IWAI et TANAKA (Ig68) qui suggèrent que les grosses gouttelettes seraient une forme de transport, les présentes observations nous conduisent à les interpréter chez la Truite comme une forme de stockage temporaire d'acides gras à l'état estérifié, la forme d'exportation des acides gras étant représentée par les triglycérides des chylomicrons.

Chez les Mammifères, la plus grande activité des enzymes intervenant dans l'estérification des acides gras a été trouvée dans la fraction microsomale de la cellule absorbante (ISSEIBACHER, I967; BickerstAFFE et ANNison, I969). Ces enzymes se localiseraient dans les membranes du reticulum (SJöstrand et BorGstrëm, I967). Pour d'autres, cependant, l'estérification s'effectuerait dès la pénétration dans la cellule absorbante, au niveau de la membrane plasmique (RoBrns, SMal, et DoNAL,DSON, I969).

Chez la Truite, la présence d'une quantité importante de triglycérides à l'extérieur du reticulum, sans que le fonctionnement de ce dernier paraîsse pertubé, incite à proposer l'alternative suivante :

I. Les triglycérides des LE sont synthétisés par le même système enzymatique que celui des chylomicrons, cette synthèse ayant lieu probablement sur la face externe de la membrane réticulaire. On voit mal en effet comment les triglycérides pourraient quitter la lumière du reticulum une fois qu'ils ont commencé à s'accumuler pour former les chylomicrons.

2. Les triglycérides des LE sont synthétisés par un système enzymatique distinct de celui des triglycérides des chylomicrons, et non lié au reticulum.

Il serait intéressant de rechercher les acides gras dont 1'administration provoque l'apparition des $L_{E} E$ et d'étudier l'effet d'une inhibition de la synthèse protéique sur l'absorption, d'une part des acides gras qui forment des LE et, d'autre part, des acides gras qui n'en forment pas.

Rę̧u pour publication en avril $19 \% 0$.

\section{SUMMARY}

FORM AND PATHWAY OF LONG CHAIN FATTYY ACID IN'TESTINAI, ABSORPTION

IN RAINBOW TROUT. II. - " STELLATE LIPIDS "

Oleic acid absorption in the intestinal mucosa of rainbow trout, acclimatized at two temperature levels $\left(7^{\circ} \mathrm{C}\right.$ and $\left.22^{\circ} \mathrm{C}\right)$ was studied by light and electron microscopy. In the epithelial absorptive cells, lipid was present with abundance in a different form from chylomicra, corresponding with irregular masses of fat or free lipid droplets previously described in mammals. These triglyceride accumulations did not appear to be enclosed by any cytomembrane and were absent from intercellular spaces.

They were observed at the two temperature levels studied in fasted or fed animals and were also present after feeding linoleic acid but not after stearic acid was given.

These stellate lipids were interpreted as temporary storage forms of fatty acid and are certainly not exported as such. It is proposed that the absorptive intestinal cells of the rainbow trout possess an esterifying mechanism, as well as other cell types such as hepatocyte or adipocyte and that the "stellate " lipids are the result neither of the inhibition of chylomicra formation. nor of the chylomicra fusion. 


\section{RÉFÉRENCES BIBLIOGRAPHIQUES}

Bergot P., Fléchon J.-E., r97o. Forme et voie d'absorption intestinale des acides gras à rhaine longue chez la truite arc-en-ciel (Salmo goirdnerii Rich). I. Lipides ent particules. Ann. Biol. anim. Biochim. Biophys. (sous presse).

Bergot P., Vodovar N., I967. Absorption des acides gras par la truite arc-en-ciel (Salmo gairdnerii RICH). C. R. Acad. Sci. Ser. D, 265, I $530-1532$.

Bickerstaffe R., Annison F. F., I969. Triglyceride synthesis by the small intestinal epithelium of the pig, sheep and chicken. Biochem. J. 111, 4r9-429.

Cardell R. R., Sadenhausen S., Porter K. R., I967. Intestinal absorption in the rat. An electron microscopic study. J. Cell Biol., 34, I23-156.

Dobbins W. O., I966. An ultrastructural study of the intestinal mucosa in congenital lipoprotein deficiency with particular emphasis upon the intestinal absortive cell. Gastroenterologv, 50, I95-210.

Fawcert D. W., 1964, in Meng H. C. Proceedings of an International Symposium on Lipids Transpor 2 I0-237. C. C. Thomas, Springfield.

FAwcetT D. W., I966. An atlas of fine structure. The cell, its organelles and inclusions. W. B. Saunders Co, Philadelphia and London.

Holmax J., I968. Ultrastructure of globule leucocytes in the lipids resorbing intestinal epithelium of the chick. Acta Univ. Agric., 37, 193-197.

Isselbacher K. J., I967. Biochemical aspects of lipid malabsorption, Fed. Proc., 26, r420-I425.

IwAI T., r968. The comparative study of the digestive tract of Teleost larvae. V. Fat absorption in the gut epithelium of goldfish larvae. Bull. jap. Soc. Scient. Fish., 34, 973-978.

Iwai T., Tanaka M., I968. The comparative study of the digestive tract of Teleost larvae. IV. Absorption of fat by the gut of the halfbeak larvae. Bull. jap. Soc. scient. Fish., 34, 871-875.

Korn E. O., 1968. Structure and function of the plasma membrane. A biochemical perspective. J. gen. Physiol., 52, 257s-278s.

PAlay S., Revel J., I964. The morphology of fat transport. In : Meng H. C. Proceedings of an inter. national symposium on lipid transport, 33-69, C. C. Thomas, Springfield.

Riemersma J. C., rg68. Osmium tetroxyde fixation of lipids for electron microscopy. A possible reaction mechanism. Biochim. Biophys. Acta, 152, 718-727.

Robins S. J., Small, D. M., et Donaldson R. M., 1969. Triglyceride formation in intestinal microvillous membrane during fat absorption. J. clin. Invest., 48, $69 \mathrm{a}$.

Sabesin S. M., Isselbacher K. J., 1965. Protein synthesis inhibition mechanism for the production of impaired fat absorption. Science, 14\%, II49-II5I.

SсніеснL H., I968. Einige chemische Aspekte der Osmiumtetroxidfixierung. Zeitsch. Naturforsch., 23, $989-992$.

Schoefl G. I., I968. The ultrastructure of chylomicra of the particles in an artifical fat emulsion. Proc. R. Soc. B.,169, I47-I52.

Sjöstrand F. S., Borgström B., 1967. The lipid components of the smooth surfaced membrane bounded vesicles of the columnar cel's of the rat intestinal epithelium during fat absorption. J. Ultrastruct. Res., 20, I40-160.

Spencer R. P., Schetg R. I., Binder H. J., 1966. Observations on lipids of the alimentary canal of the Hagfish, Eptatretus stoutii. Comp. Biochem. Physiol., 19, I39-I44.

TAYlor A. B., Adamstone F. B., 1965. Fat absorption and transport in intestinal cell of the rat. Anat. Rec., 151, 477-478.

Trotter N., I967. Electron opaque bodies and fat droplets in mouse liver after fasting or glucose injection. J. Cell Biol. 34, 703-7II.

Vodovar N., Flanzy J., I966. Mécanisme de la pénétration et voie d'acheminement des graisses dans la cellule absorbante de l'intestin grêle du porc. Annls. Biol. anim. Biochim. Biophys., 6, 315-332.

Vodovar N., Flanzy J., Françors A. C., I967. Répartition des acides gras ingérés entre vaisseaux sanguins et lactéales centrales dans le stroma des villosités. Annls. Biol. anim. Biochim. Biophys., 7, 423-435.

Vodovar N., Flanzy J., Françors A. C., I969. Lieu de la formation des chylomicrons : leur impotance pour l'absorption normale des acides gras à chaîne longue. Aspect morphologique. Annls. Biol. anim. Biochim. Biophys, , 9, 219-232.

Woop E. M., I967. An ordered complex of filaments surrounding the lipid droplets in developing adipose cells. Anat. Rec., 157, 437-447. 


\title{
ABRÉVIATIONS DES LÉGENDES DES PLANCHES
}

\author{
D desmosome \\ EI espace intercellulaire \\ EPN espace péri-nucléaire \\ F filaments \\ $\mathrm{G}$ golgi \\ LE lipides étalés \\ LP particules lipidiques (chylomicrons) \\ M mitochondrie \\ MP membrane plasmique \\ $\mathrm{N}$ noyau \\ RE réticulum endoplasmique \\ S stroma \\ SL structure lamellaire \\ $\mathrm{ZA}$ zone apicale
}




\title{
PLANCHE, I
}

\author{
FIG. I
}

Lipides " étalés 》 (LE) dans la partie supranucléaire des cellules épithéliales absorbantes. Présence de chylomicrons (L,P) dans le RE, dans l'appareil de Golgi (G) et les espaces intercellulaires.

Truite acclimatée à $7^{\circ} \mathrm{C}, 8 \mathrm{~h}$ après administration d'acide oléique. Double fixation $\mathrm{OsO}_{4}$. Double coloration ( $\left.\times 39000\right)$. 


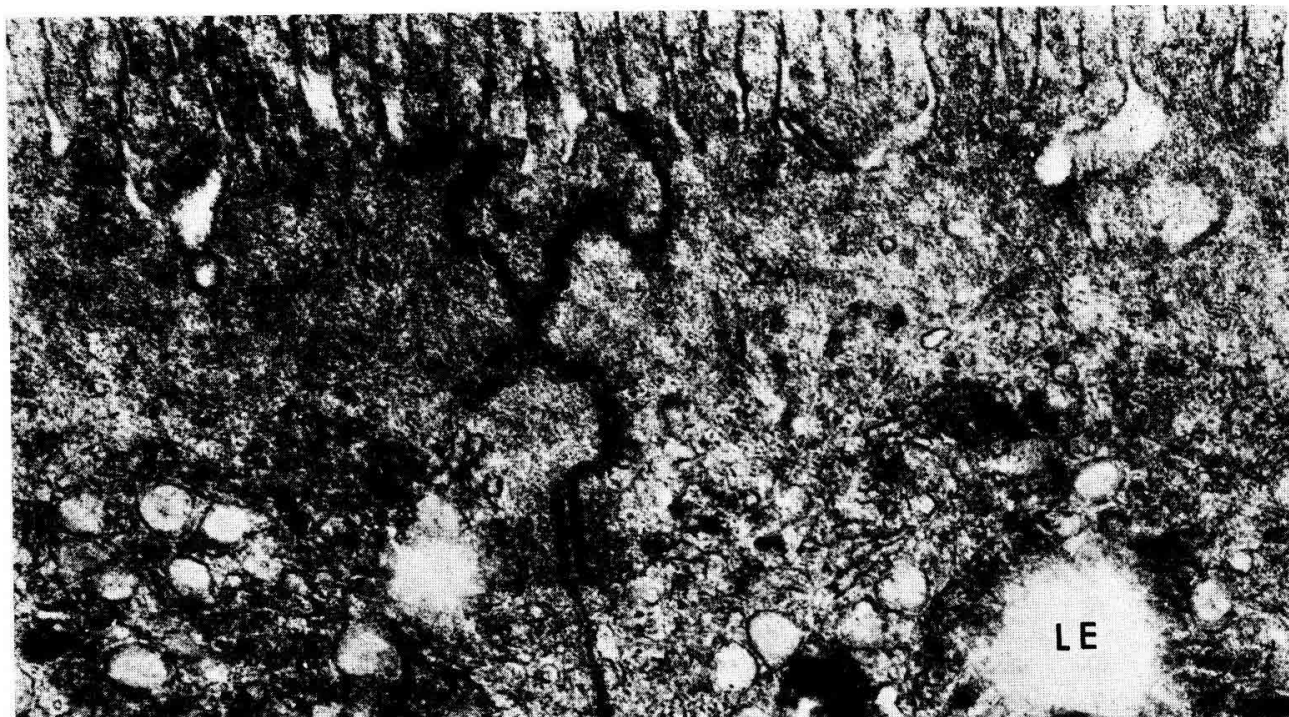

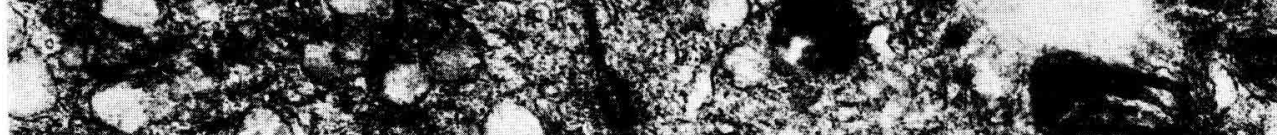
7.5.

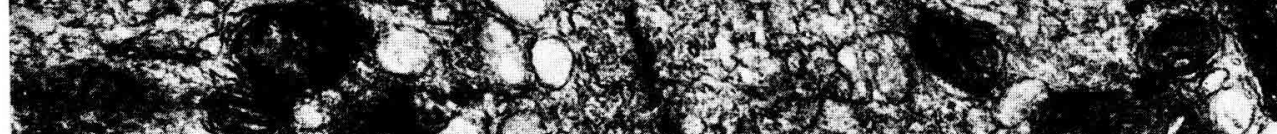

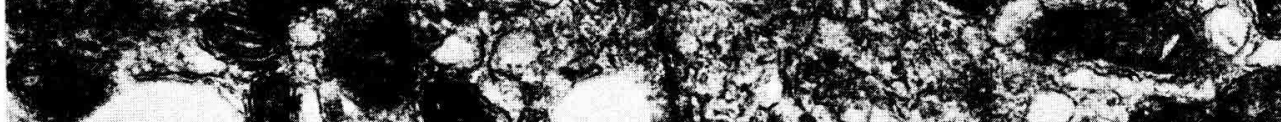
6I LE

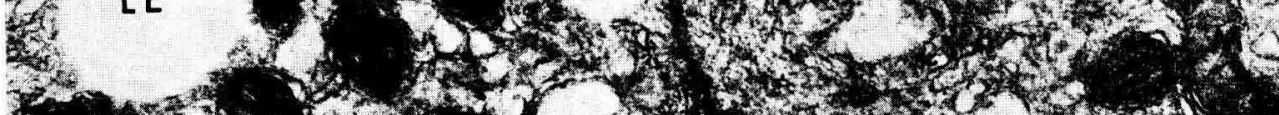

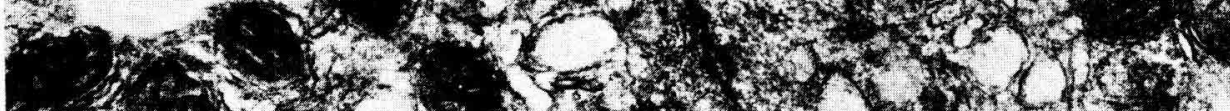

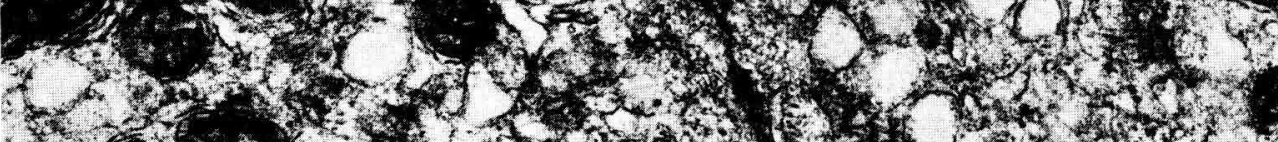

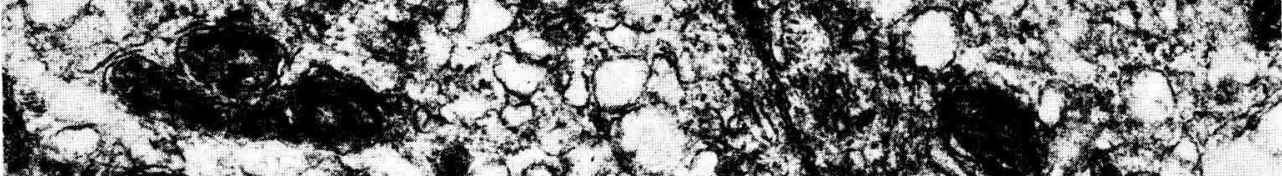
36.

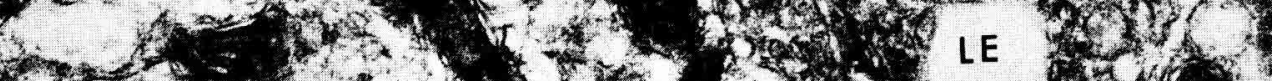

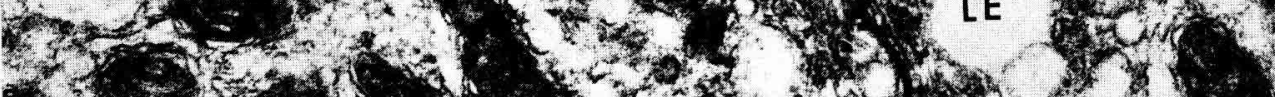

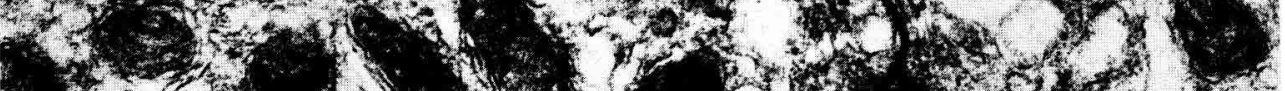

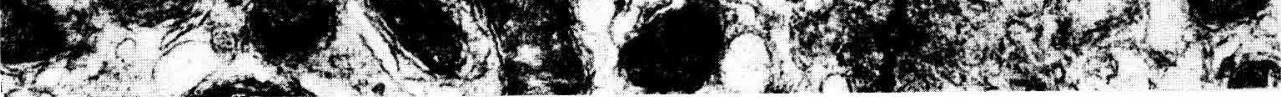

P. BERGOT, J.-E. FLÉCHON 
PLANCHE II

\section{FIG. 2}

Truite acclimatée à $22^{\circ} \mathrm{C}, 8 \mathrm{~h}$ après administration d'acide oléique. Fixation $\mathrm{OsO}_{4}$. Double coloration.

Accumulation de lipides "étalés " (LE). La bordure dense qui les entoure est visible au niveau des LE parvenus en contact.

Présence de chylomicrons (LP) dans le reticulum endoplasmique et l'espace intercellulaire.

X 22500. 

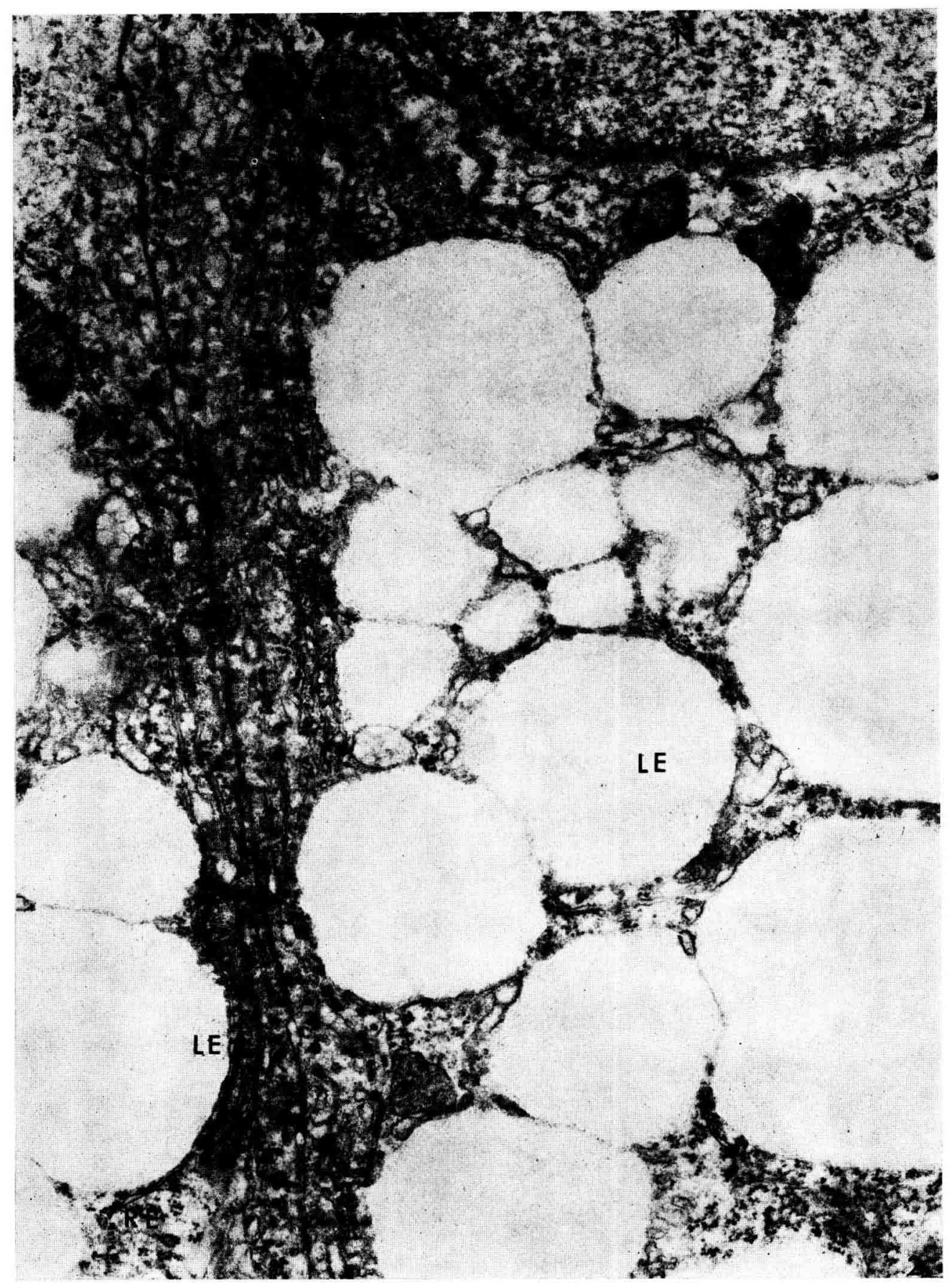

P. BERGOT, J.-E. FLÉCHON 


\section{PLANCHE III}

Présence simultanée de lipides étalés (LL) dans le cytoplasme et de chylomicrons (LP) dans le reticulum endoplasmique et l'espace périnucléaire de la cellule absorbante. Truite acclimatée à $7^{\circ} \mathrm{C}$.

Fig. 3

8 h après administration d'acide linoléique Fixation $\mathrm{OsO}_{4}$. Double coloration.

$$
\text { × } 35000 \text {. }
$$

Fig. 4

$8 \mathrm{~h}$ après administration d'acide oléique.

Double Fixation. Double coloration.

Fig. 5

$16 \mathrm{~h}$ après administration d'acide oléique. Fixation $\mathrm{OsO}_{4}$. Double coloration.

$\times 64000$.

La bordure dense des LE apparaìt bien différente des membranes cytoplasmiques (reticulum endoplasmique et enveloppe nucléaire) et des membranes plasmiques (membrane plasmique latérale et membrane des structures lamellaires). 
r.j. $3 x^{3} x^{2}+8$

$-\frac{13}{2}+2$

$-1 \%$

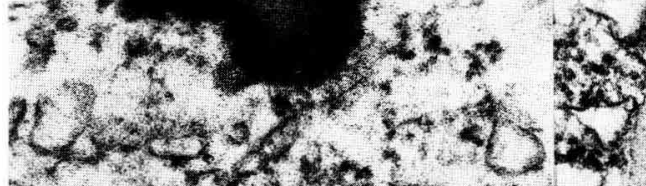

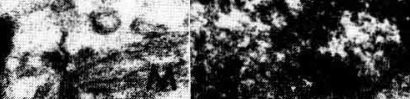

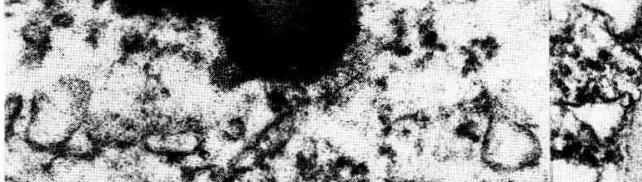

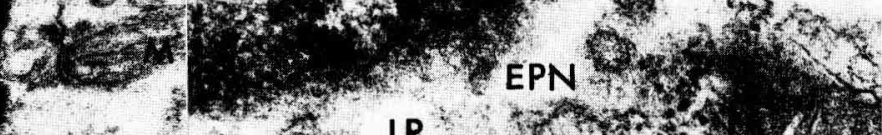

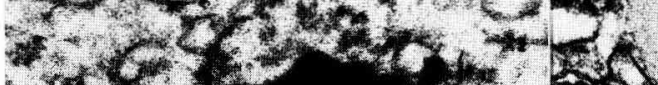

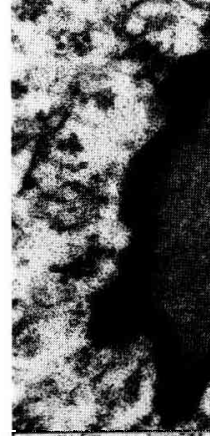
$2 x^{2}+x^{2}+3 x$

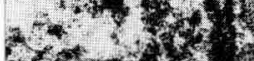

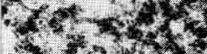

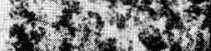

$30 \sin 20$

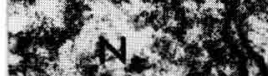

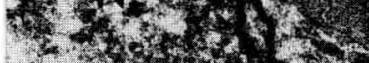

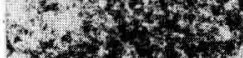

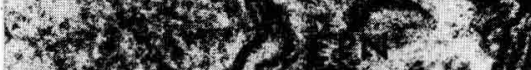

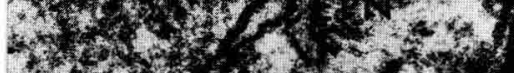

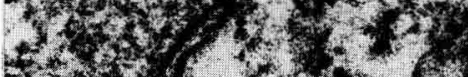

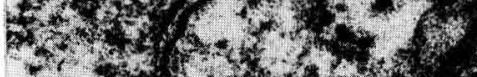

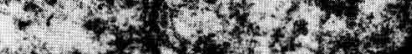

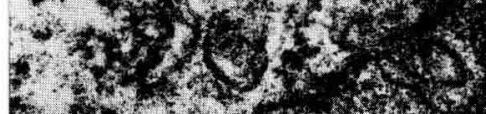

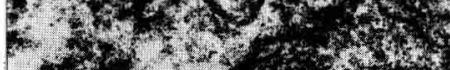

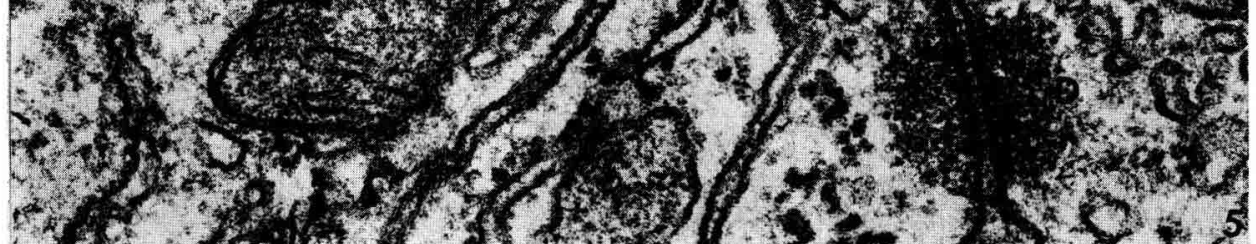

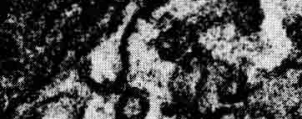
$x^{2}+w^{3}=2$

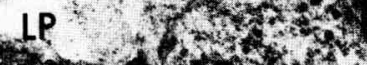

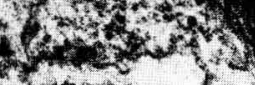
fow 1.9.8

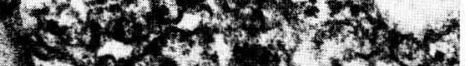

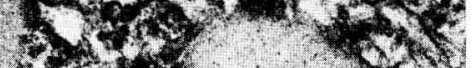
gf $2 x$

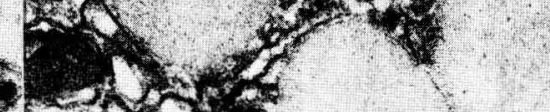

$\frac{6}{3+1}$ and $(20)$ LE

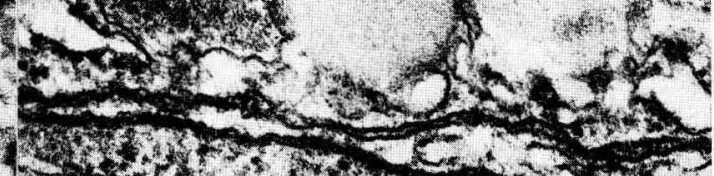
(3) 3 in $\operatorname{loc}^{2}$ t

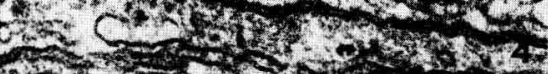

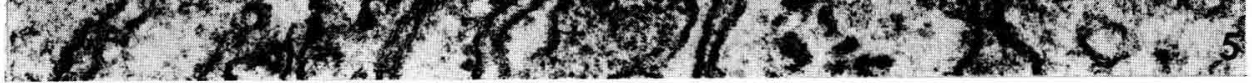


PLANCHE IV

Truite acclimatée à $2{ }^{\circ} \mathrm{C}, 16 \mathrm{~h}$ après administration d'acide oléique. Fixation $\mathrm{OsO}_{4}$. Double coloration.

FIG. 6

Lipides étalés (LE) dans le cytoplasme infranucléaire de la cellule absorbante. Une des masses lipidiques parait partiellement bordée par des filaments (F). 


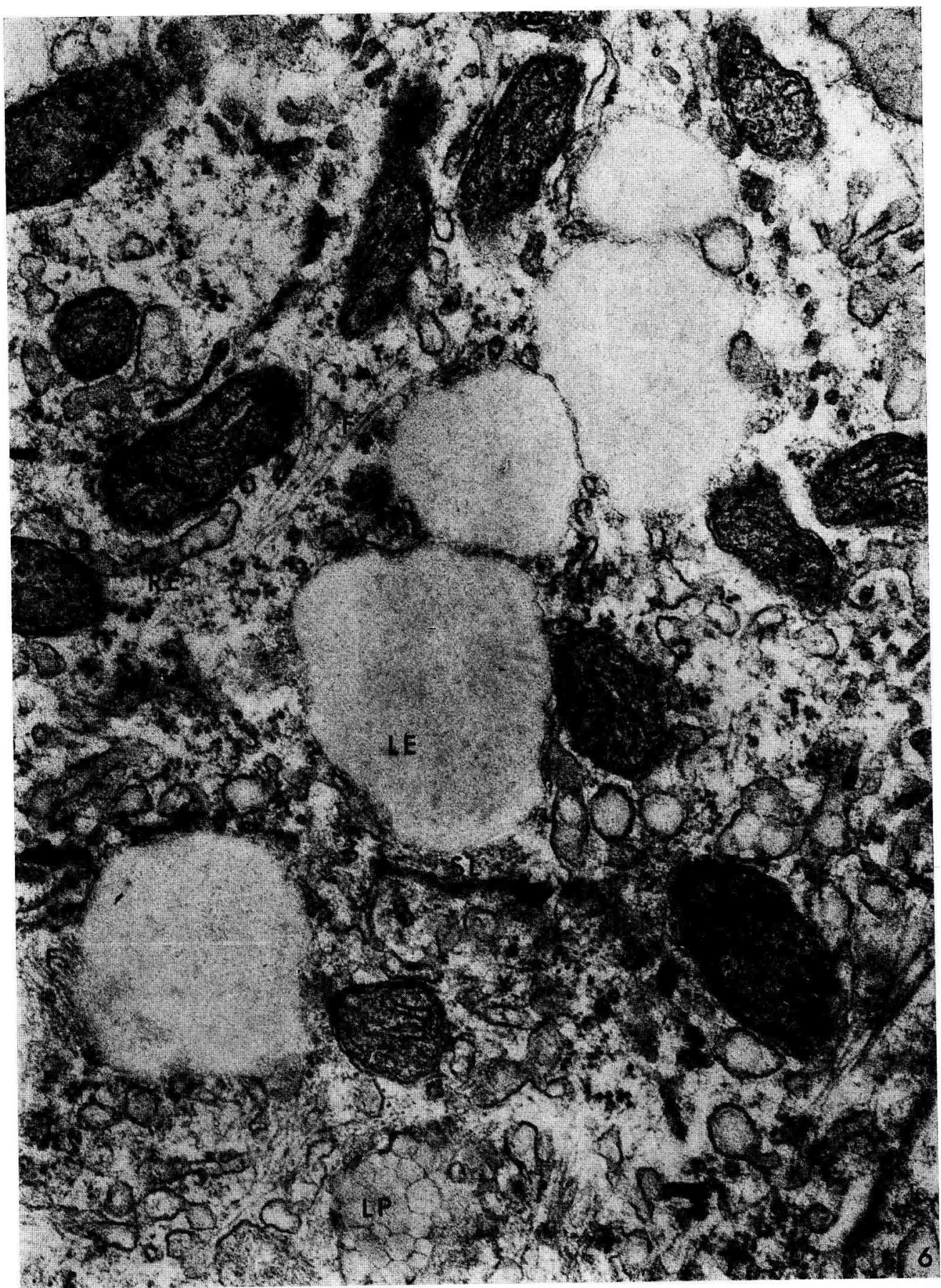

P. BERGO'T, J.-E. FLÉCHON 